\title{
Multiple large xanthomas: A case report
}

\author{
$\mathrm{CHEN} \mathrm{ZHAO}^{1 *}$, MINGXIANG KONG $^{2 *}, \mathrm{LICAO}^{1}$, QIONG ZHANG $^{3}$, YONG FANG $^{2}$, \\ WEIWEI RUAN ${ }^{2}$, XIAOFAN DOU ${ }^{1}$, XIAOHUI GU ${ }^{1}$ and QING BI $^{2}$
}

\author{
${ }^{1}$ Department of Orthopedics and Joint Surgery, Zhejiang Provincial People's Hospital, Hangzhou, Zhejiang 310014; \\ ${ }^{2}$ Department of Orthopedics, The Second Clinical Medical College, Wenzhou Medical University, Wenzhou, Zhejiang 325000; \\ ${ }^{3}$ Operating Room, Zhejiang Provincial People's Hospital, Hangzhou, Zhejiang 310014, P.R. China
}

Received March 8, 2015; Accepted March 22, 2016

\section{DOI: $10.3892 / \mathrm{ol} .2016 .5282$}

\begin{abstract}
A 23-year-old male patient presented with multiple large masses in his elbows, buttocks, knees, Achilles tendons, feet, shoulders and hands. The large masses in the elbows and buttocks measured $\sim 6 \times 5 \times 5 \mathrm{~cm}$ and $\sim 7 \times 5 \times 4 \mathrm{~cm}$, respectively. The patient presented with an elevated level of low-density lipoprotein cholesterol, and had been previously diagnosed with homozygous familial hypercholesterolemia (FH) and multiple xanthomas. Local surgical excisions were performed to remove the massive xanthomas from the elbows and buttocks, and histological analysis of the surgical specimens confirmed the previous diagnosis of homozygous $\mathrm{FH}(\mathrm{HoFH})$. The aim of the present study was to report a rare case of HoFH coinciding with multiple, large and widely-distributed xanthomas and to discuss the clinical characteristics, in order to provide a better understanding of xanthomas and FH.
\end{abstract}

\section{Introduction}

A xanthoma refers to an exogenous mass that is visible on the body surface. Xanthomas are not tumors but clusters of foam cells that form within the connective tissue of the skin, tendons and subcutaneous tissues. Xanthomas comprise important clinical manifestations of lipid metabolism disorders, and are commonly encountered in patients with familial hypercholesterolemia (FH) (1). Xanthomas may be divided into several categories: Tendinous xanthoma, xanthoma tuberosum, eruptive xanthoma, xanthoma planum and palmar xanthoma (1). The most commonly observed xanthomas among patients with FH are tendinous xanthomas (40-50\% of all patients), which are subcutaneous tumors located within the tendons used for extension, and mainly affect the Achilles, patellar tendons and

Correspondence to: Dr Qing Bi, Department of Orthopedics, The Second Clinical Medical College, Wenzhou Medical University, 109 Xueyuan West Road, Wenzhou, Zhejiang 325000, P.R. China E-mail: 13588302991@126.com

${ }^{*}$ Contributed equally

Key words: xanthomas, familial hypercholesterolemia extensor tendons of the hands, buttocks, elbows, eyelids and hand creases $(2,3)$. Tuberous xanthomas are also commonly observed in FH patients (10-15\% of all patients), and manifest as yellow nodules, often measuring $\leq 2 \mathrm{~cm}$ in diameter, and are located in pressure areas, including the extensor aspects of the knees, elbows and buttocks (2-4). The clinical manifestations associated with xanthomas depend on the duration and severity of hyperlipoproteinemia (5); therefore, the presence of multiple xanthomas often indicates severe and long-term $\mathrm{FH}$ and tends to be observed in patients with homozygous $\mathrm{FH}$ $(\mathrm{HoFH})(2,6)$.

FH is an inherited disorder featuring elevated plasma levels of low-density lipoprotein cholesterol (LDLC), atherogenesis and xanthomas (7). Two clinical variants of this disorder exist, the homozygous and heterozygous variants. Heterozygous FH $(\mathrm{HeFH})$ accounts for the majority of FH cases, with an approximate incidence of 1 per 500 individuals; whereas the homozygous variant of the disease is considerably rare, with an approximate incidence of 1 per 1,000,000 individuals (3). The clinical features of $\mathrm{HeFH}$ are mild, and include a blood LDLC concentration of $>4.9 \mathrm{mmol} / \mathrm{l}$, total cholesterol (TC) levels $>7.5 \mathrm{mmol} / \mathrm{l}$ and an age of onset of $>20$ years (8). By contrast, HoFH is characterized by a greater incidence rate of cutaneous xanthomas, enlarged Achilles tendons, atherosclerosis, corneal arcus, a blood LDLC concentration of $>13 \mathrm{mmol} / \mathrm{l}$, TC levels $>14.95 \mathrm{mmol} / \mathrm{l}$ and disease onset in early childhood (7).

The present study reports the case of a 23-year-old male patient with $\mathrm{HoFH}$, who presented with multiple large tuberous and tendinous xanthomas within various dermal tissues.

\section{Case report}

A 23-year-old male patient presented at Zhejiang Provincial People's Hospital (Hangzhou, China) in May 2008 with multiple yellowish elevated masses over the dorsum of the elbows, knees, buttocks and hands. The size of the masses varied between 1x1x1 cm (over the dorsum of the hands) and $7 \times 5 \times 4 \mathrm{~cm}$ (over the dorsum of the elbows) (Fig. 1). The lesions were originally asymptomatic; they appeared at 2 years of age and then progressively increased in size and extent. The patient had symptoms of discomfort and pain in the elbows and buttocks, which were due to the large size of the masses.

The plasma TC levels of the patient were $14.95 \mathrm{mmol} / \mathrm{l}$ (reference value, 3.11-5.96 mmol/l), whereas the LDLC level 

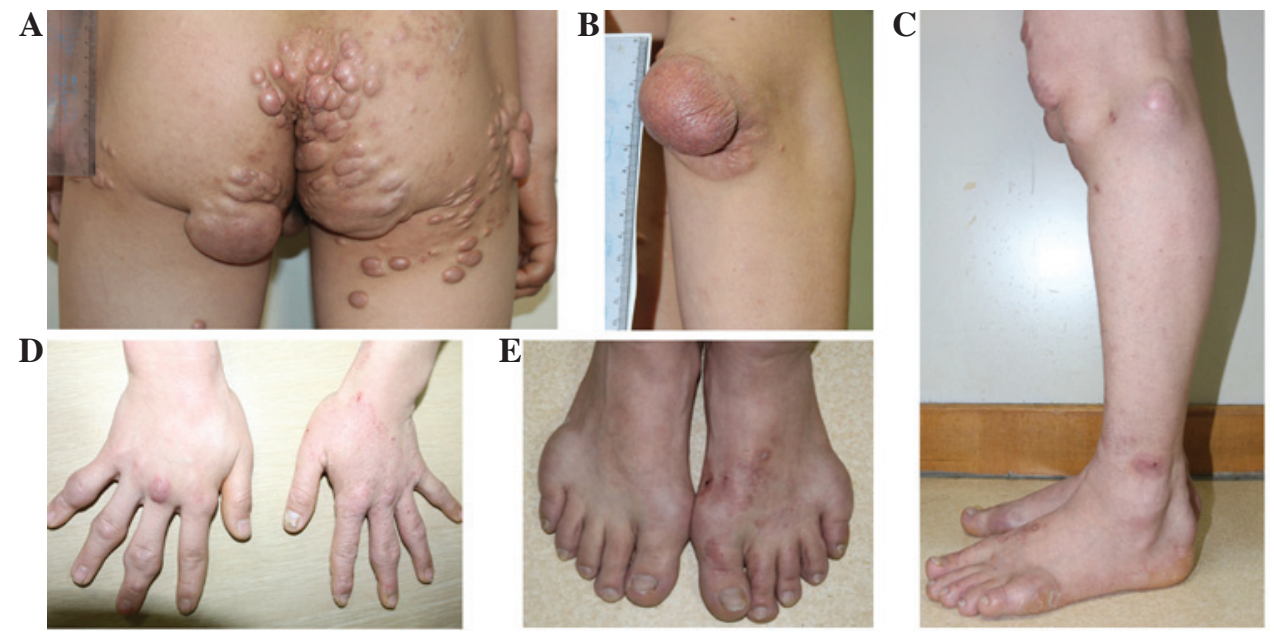

Figure 1. Multiple yellowish masses over the (A) buttocks, (B) elbows, (C) knees and ankles, (D) hands and (E) feet of the patient. Largest xanthoma of the (B) right elbow measured $\sim 6 \times 5 \times 5 \mathrm{~cm}$, whereas the largest xanthoma of the (A) buttocks measured $\sim 7 \times 5 \times 4 \mathrm{~cm}$.
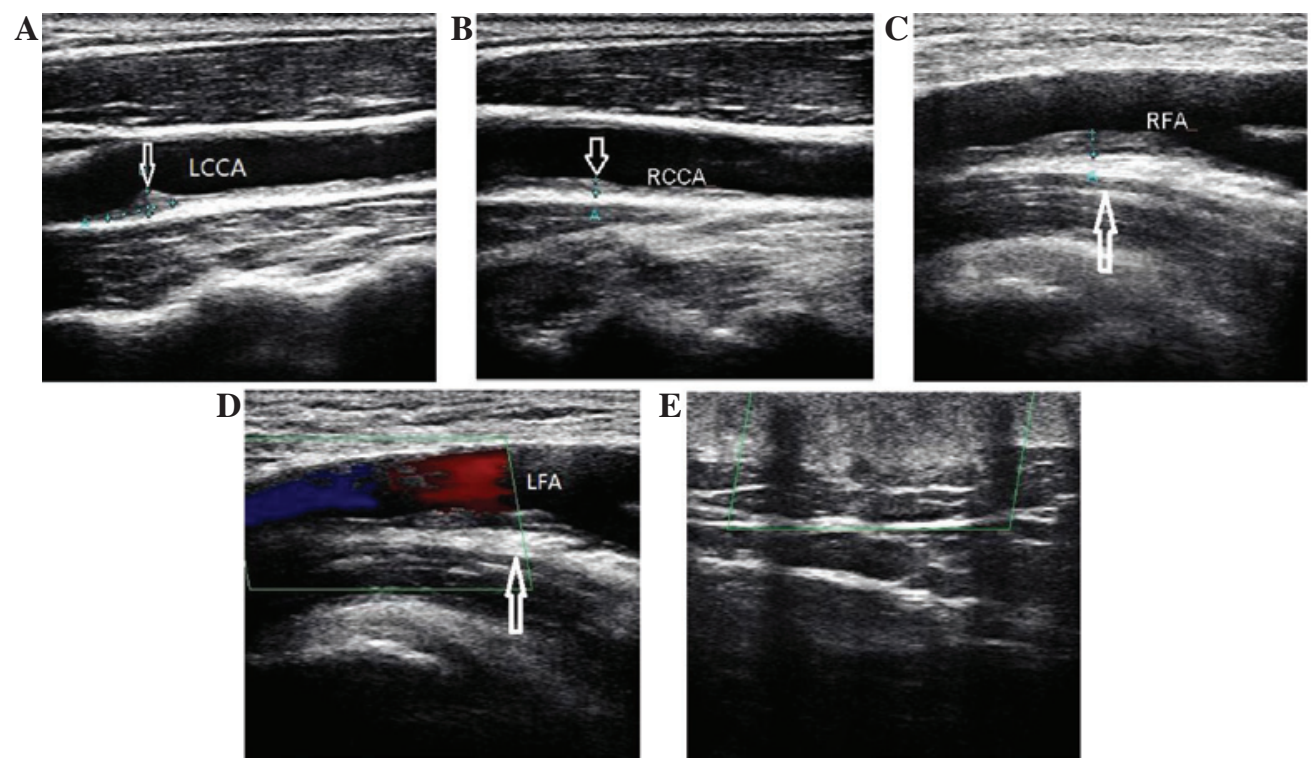

Figure 2. Doppler ultrasound scan. (A) Left carotid artery had formed atherosclerotic lesions and the intima-media was $1.0 \mathrm{~cm}$ thick, with soft plaque formation in the sinus. The plaque measured $\sim 6.5 \times 2.2 \mathrm{~mm}$. (B) Right carotid artery had formed atherosclerotic lesions and the intima-media was $1.3 \mathrm{~cm}$ thick, with soft plaque formation in the sinus. The plaque measured $\sim 6.1 \times 2.0 \mathrm{~mm}$. (C) Right common femoral artery had formed atherosclerotic lesions and the intima-media was $2.1 \mathrm{~cm}$ thick. (D) Left common femoral artery had formed atherosclerotic lesions and the intima-media was $2.5 \mathrm{~cm}$ thick. (E) Ultrasound images of the right elbow mass with high echo. The border of the mass was clear. Multiple hypoechoic foci were identified in the mass. Certain regions of the mass were fused, with visible blood spots and a lack of blood flow.

was $12.69 \mathrm{mmol} / \mathrm{l}$ (reference value, $2.10-3.10 \mathrm{mmol} / \mathrm{l})$. The apolipoprotein (Apo) A1 level was $0.74 \mathrm{~g} / 1$ (reference value, 1.10-1.76 g/l), the Apo B level $2.84 \mathrm{~g} / 1$ (reference value, 0.63-1.14 g/l) and the Apo A1/Apo B ratio was 3.84 (reference value, $0.40-1.96)$. Test results for liver enzymes, renal function, blood glucose, uric acid, free thyroxin and thyroid-stimulating hormone were normal. A Doppler ultrasound scan (TA700; Toshiba, Tokyo, Japan) revealed that the two carotid arteries and the lower extremity arteries presented with progressive atherosclerosis (Fig. 2). The carotid arteries had accumulated atheromatous plaques, and the lower extremity arteries had progressive calcific sclerosis. Patient chest X-ray, abdominal ultrasound, electrocardiogram and echocardiogram were normal. Magnetic resonance imaging (MRI) scans (Siemens AG, Munich, Germany) revealed decreased signal intensity on
T1-weighted and T2-weighted spin-echo images of the masses in the elbows, knees and posterior malleolus. Xanthomas had infiltrated the triceps tendon and the patellar ligament. MRI scan also revealed a thickened Achilles tendon and patellar ligament (Fig. 3). Neurological examination for central nervous system function and brain MRI scan were performed to determine the presence or absence of cerebrotendinous xanthomatosis (9), a rare autosomal-recessive familial lipid metabolic disease caused by mutations in sterol 27 hydroxylase; however, no abnormality was identified.

The father of the patient had a history of coronary heart disease (CHD) and was treated with percutaneous coronary intervention 2 years previously. The mother and sister had no reported symptoms of CHD or similar skin lesions. The two parents demonstrated elevated TC and LDLC levels, a typical 
Table I. Lipid profiles of the patient, the patient's parents and the patient's sister.

\begin{tabular}{lccccc}
\hline & & & \multicolumn{2}{c}{ Cholesterol } & \\
Individual & Age, years & $\mathrm{TC}, \mathrm{mmol} / \mathrm{l}$ & $\mathrm{HDL}, \mathrm{mmol} / \mathrm{l}$ & $\mathrm{LDL}, \mathrm{mmol} / \mathrm{l}$ & $\mathrm{TG}, \mathrm{mmol} / \mathrm{l}$ \\
\hline Patient & 23 & 14.95 & 1.90 & 12.69 & 1.06 \\
Father & 57 & 7.18 & 1.13 & 5.41 & 0.68 \\
Mother & 49 & 7.53 & 1.14 & 6.65 & 0.69 \\
Sister & 27 & 3.92 & 1.34 & 2.38 & 0.56 \\
Normal range & & $3.11-5.96$ & $1.04-2.05$ & $2.10-3.10$ & $0.34-1.69$ \\
\hline
\end{tabular}

TC, total cholesterol; TG, triglycerides; HDL, high-density lipoprotein; LDL, low-density lipoprotein.
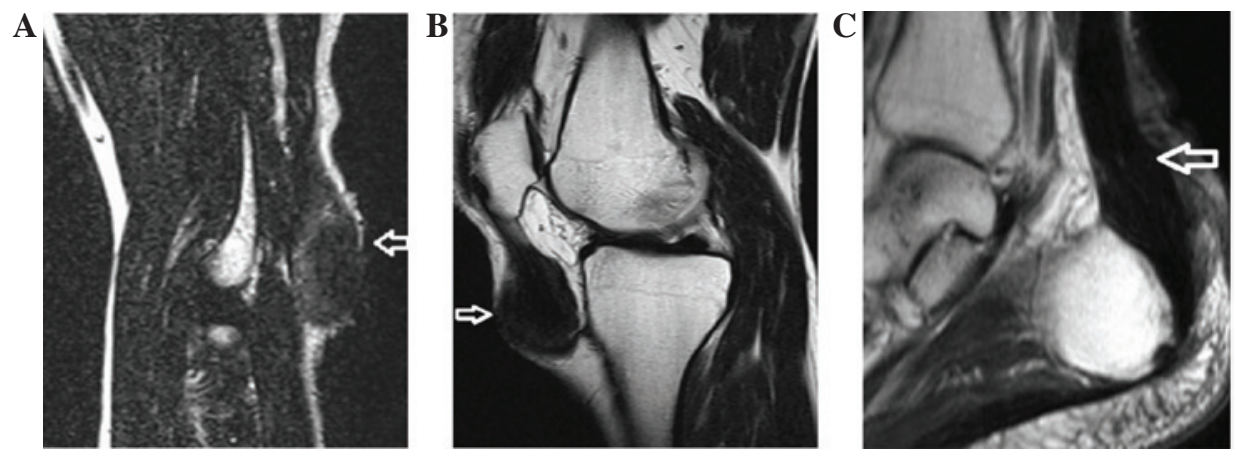

Figure 3. Magnetic resonance imaging scan of the left elbow, left knee and left Achilles tendon. (A) A mass that had infiltrated the triceps tendon was identified in the left elbow; (B) a mass that had infiltrated the patellar ligament was identified in the left knee; and (C) a thickened Achilles tendon was observed. Arrows show the position of the xantomas tumors.
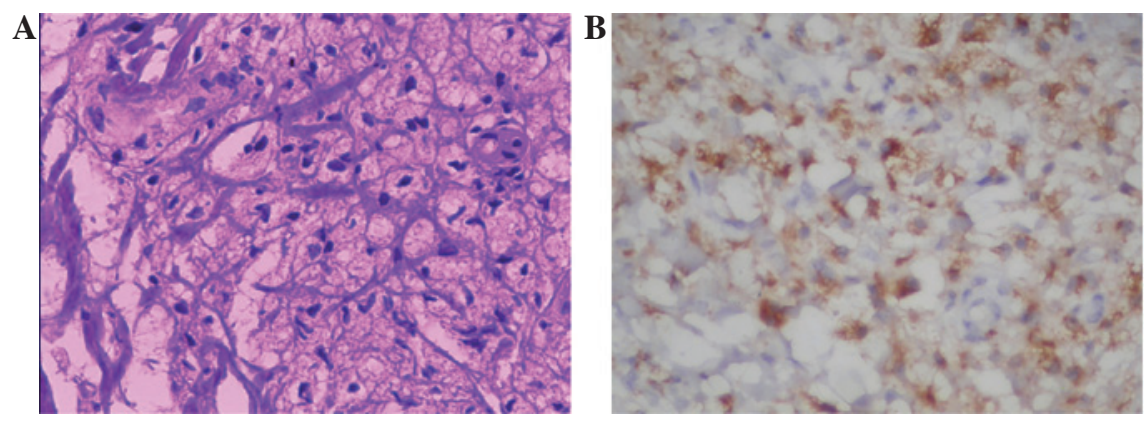

Figure 4. (A) Light photomicrograph of a xanthoma specimen showing foamy macrophages with fibrosis and cholesterol clefts (hematoxylin and eosin stain; original magnification, x400). (B) Light photomicrograph of a xanthoma specimen showing that the foamy cells strongly expressed cluster of differentiation 68 (immunohistochemistry; original magnification, $\mathrm{x} 100$ ).

characteristic of $\mathrm{HeFH}$, whereas the sister did not. Secondary hypercholesterolemia, which may be caused by hypothyroidism, diabetes and renal or hepatic disease, were excluded. The lipid profiles of the patient and his family are described in Table I.

The patient was diagnosed with $\mathrm{HoFH}$ and multiple xanthomas. The patient subsequently abstained from alcohol and tobacco use, and followed a vegetarian diet that was low in saturated fat and cholesterol, as advised by physicians. In addition, the patient was treated with a combined treatment regimen of rosuvastatin $(10 \mathrm{mg} /$ day $)$ and ezetimibe $(10 \mathrm{mg} /$ day) for a year. Following a year of conservative treatment, no marked decrease in the plasma TC levels (controlled at $13.05 \mathrm{mmol} / \mathrm{l}$ ) or substantial regression of the multiple xanthomas was observed. Due to the symptomatic nature of the lesions and for cosmetic reasons, surgical removal of the large xanthomas was considered necessary. Surgical excision of the masses over the elbows and buttocks was performed. Intraoperatively, the yellowish xanthomatous masses in each elbow were found to closely adhere to the triceps tendon, which prevented the complete excision of these lesions. Excision was not performed for all lesions, since the extent of xanthomatous involvement had not been clearly determined. Resected tissues were sent to the Department of Pathology, Zhejiang Provincial People's Hospital for tissue examination. Microscopic examination of the surgical specimens (Olympus 
BX50; Olympus Corporation, Tokyo, Japan) revealed nests composed of xanthoma tissue, which consisted of connective tissue and foam cells, containing cholesterol, cholesterol esters, triglycerides and phospholipids (Fig. 4A). These findings confirmed the diagnosis of HoFH. Immunohistochemical staining showed that the foam cells strongly expressed cluster of differentiation 68 (CD68) (Fig. 4B). Treatment with rosuvastatin and ezetimibe was continued postoperatively, and the dose of rosuvastatin was adjusted to $20 \mathrm{mg} /$ day. Four weeks subsequent to surgery, the symptoms had largely resolved. At that point, apheresis therapy was recommended for a better control of the high LDLC levels, but the patient declined due to the high cost of the procedure. Although the patient had no symptoms of CHD, he was advised to take note of any chest tightness and other symptoms of CHD and undergo a cardiac examination at least twice a year. One year following surgery, the serum LDLC levels of the patient were controlled at $8.50 \mathrm{mmol} / 1$ with no symptoms of CHD or postoperative recurrence of xanthomas.

Written informed consent was obtained from the patient for the publication of this study, and the study was approved by the Ethics Review Committee of Zhejiang Provincial People's Hospital.

\section{Discussion}

Xanthomas are palpable masses that are typically located within the skin or subcutaneous tissue and consist of cholesterol, cholesterol esters, triglycerides, phospholipids, and numerous lipid-laden foamy macrophages (10). Xanthomas have been indicated to commonly occur in patients with FH (1). A previous study has shown that the most frequent site for xanthomas is the Achilles tendon (11). Other frequent sites include the extensor tendons of the hands and feet, as the extensor tendon areas are subject to mechanical stress (11). The mechanical stress encountered in tendons is similar to recurrent trauma, which is thought to predispose these sites to the development of xanthomas $(12)$. Courtice $(13,14)$ indicated that the concentration of LDLs increased in the lymphatic drainage system of a rabbit paw that was subjected to mild heat injury of the skin. Courtice also argued that increased capillary permeability to various subclasses of LDL resulted in an increased extravascular LDL concentration, and that a portion of the extracellular LDL may move into the lymphatic system, which is responsible for draining the area of the paw. Mechanical stress, similarly to the effect of mild heat injury to the skin, also increases capillary permeability around the mechanical stress-exposed areas, which may lead to the accumulation of LDLC. This theory may explain why tuberous xanthomas are predominantly encountered in mechanical stress-exposed regions.

The present patient presented with multiple xanthomas in the mechanical stress-exposed parts of the body, including the buttocks, extensor aspect of the knee, elbow and ankle. The occurrence of multiple large xanthomas is rare. Although the appearance is similar to tumors, xanthomas have distinct characteristics that allow for the differentiation of the two. In the majority of cases, xanthomas are asymptomatic; unless they grow to be large in size and cause compression to adjacent structures, which may cause pain and mobility problems.

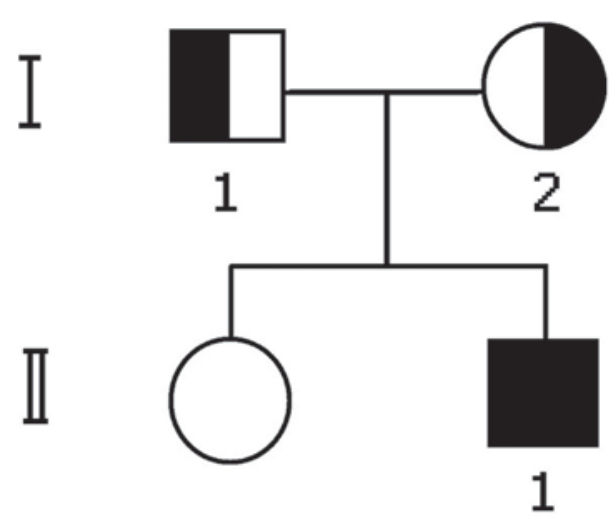

Figure 5. Pedigree of the proband. Square symbols are male and circle symbols are female. Half-filled symbols indicate heterozygous FH, presenting with mildly elevated levels of low-density lipoprotein cholesterol in the blood, and represent the parents of the patient. Completely filled (black) symbol indicates homozygous FH, and represents the proband. Empty symbol (white) indicates unaffected family members, and represents the sister of the proband. FH, familial hypercholesterolemia.

Histologically, xanthomas consist of foamy macrophages with fibrosis and cholesterol clefts. A high level of LDLC is the most common clinical manifestation and the main cause of xanthomas in patients with FH. In these patients, markedly elevated LDLC levels are secondary to LDL receptor defects and result in lipid leakage from the vasculature into the surrounding tissue. This results in the uptake of lipids by macrophages, which, in turn, leads to the increased accumulation of undegraded lipids and formation of foam cells $(1,15)$. Extracellular cholesterol crystallizes into clefts, inducing an inflammatory reaction with giant cells and resulting in fibrosis. CD68 is particularly useful as a marker for the various cells of the macrophage lineage, including monocytes, histiocytes, giant cells, Kupffer cells and osteoclasts (16). In addition, foam cells, which are widely prevalent in xanthomas, are strongly immunopositive for CD68 (16).

Numerous types of imaging examinations aid the diagnosis of xanthomas. Sonography is a simple, widely available and economical modality used for the identification of xanthomatosis, rendering it superior to gross clinical assessment. Sonography has been reported as an effective method for the investigation of tendinous xanthomas $(17,18)$. In the present case, Doppler ultrasound revealed multiple hypoechoic foci in the masses of each elbow.

An MRI scan is a practical imaging modality that distinguishes xanthomas from tumors. On an MRI scan, xanthomas exhibit morphological and signal intensity abnormalities. In the presence of a xanthoma, the normally flat or concave margins of tendons or other normal tissues may change and appear convex on axial MR images (11). Furthermore, tendons with xanthomas tend to a have increased signal intensity on T1- and T2-weighted spin-echo images compared with normal tendons (19). Tumors often exhibit a marked increase in signal intensity compared with xanthomas.

The patient in the present study presented with multiple large xanthomas with a wide-ranging distribution, and an onset at 2 years of age. The patient had an LDLC level of $12.69 \mathrm{mmol} / \mathrm{l}$, suggesting a high likelihood of HoFH. The parents of the patient had mildly elevated levels of LDLC 
(father, $5.41 \mathrm{mmol} / \mathrm{l}$; mother, $6.45 \mathrm{mmol} / \mathrm{l}$ ), which, when combined with the absence of xanthomas, suggests that the parents suffered from $\mathrm{HeFH}$. The patient was the offspring of two parents with $\mathrm{HeFH}$, and appeared to have inherited an HoFH phenotype associated with an increased level of serum LDLC and more severe symptoms than the parents (Fig. 5).

A high level of plasma LDLC is a risk factor for atherosclerosis. Tendinous and tuberous xanthomas may indicate $\mathrm{FH}$ and increased cardiovascular risk, as xanthomas are associated with elevated plasma concentrations of LDLC. The presence of xanthomas increases the risk of cardiovascular disease in patients with FH by as much as three-fold, indicating that xanthomas and atherosclerosis may share a certain etiology $(20,21)$. However, cardiovascular disease caused by atherosclerosis in individuals with $\mathrm{HoFH}$ typically appears during childhood and may result in mortality by 20-30 years of age (3). Measuring the intima-media thickness by ultrasound aids the identification of the preclinical stages of atherosclerosis (22). In addition, large-scale epidemiological studies have shown an association between increased intima-media thickness and future CHD and stroke $(22,23)$. In the present case, ultrasound showed an increase in the intima-media thickness of the bilateral internal carotid and femoral arteries, indicating $\mathrm{FH}$, and a high risk of developing cardiovascular disease.

The management of patients with HoFH poses a medical challenge. If left untreated, and occasionally despite maximum medical therapy, HoFH often rapidly leads to atherosclerotic changes that cause aortic stenosis and coronary artery disease (24). The first step for the treatment of HoFH is a change in lifestyle and diet regulation. The diet of $\mathrm{HoFH}$ patients must be low in saturated fats and cholesterol $(8,25)$. Combined drug therapy, including statins plus ezetimibe or bile acid resin, is recommended for patients with severe hypercholesterolemia $(1,26)$. Studies have shown that statins have the ability to soften xanthomas (1,27). Massive xanthomas may require surgical intervention (28). Surgery is only suggested for xanthomas that are extremely large and painful and cause mobility problems. Certain studies, however, have reported a high postoperative recurrence rate of xanthomas (29). Postoperative cholesterol-lowering therapy may reduce the likelihood of recurrence (27); therefore, for multiple, massive tendinous and tuberous xanthomas, local surgical excision combined with postoperative cholesterol-lowering therapy appears to be the most effective treatment option.

\section{Acknowledgements}

The present study was supported by a grant awarded to Professor Qing Bi by the Medical Science and Technology Project In Zhejaing Province (grant no. Y2012ZDA003).

\section{References}

1. Zak A, Zeman M, Slaby A and Vecka M: Xanthomas: Clinical and pathophysiological relations. Biomed Pap Med Fac Univ Palacky Olomouc Czech Repub 158: 181-188, 2014.

2. Sethuraman G, Sugandhan S, Sharma G, Chandramohan K, Chandra NC, Dash SS, Komal A and Sharma VK: Familial homozygous hypercholesterolemia: Report of two patients and review of the literature. Pediatr Dermatol 24: 230-234, 2007.
3. Alves $\mathrm{C}$ and Braid Z: Homozygous familial hypercholesterolemia: Case report of a rare cause of dyslipidemia. Pediatr Endocrinol Diabetes Metab 17: 162-165, 2011.

4. Cruz PD Jr, East C and Bergstresser PR: Dermal, subcutaneous and tendon xanthomas: Diagnostic markers for specific lipoprotein disorders. J Am Acad Dermatol 19: 95-111, 1988.

5. Maher-Wiese VL, Marmer EL and Grant-Kels JM: Xanthomas and the inherited hyperlipoproteinemias in children and adolescents. Pediatr Dermatol 7: 166-173, 1990.

6. Moyle M and Tate B: Homozygous familial hypercholesterolaemia presenting with cutaneous xanthomas: Response to liver transplantation. Australas J Dermatol 45: 226-228, 2004.

7. Raal FJ and Santos RD: Homozygous familial hypercholesterolemia: Current perspectives on diagnosis and treatment. Atherosclerosis 223: 262-268, 2012.

8. Poustie VJ and Rutherford P: Dietary treatment for familial hypercholesterolaemia. Cochrane Database Syst Rev 2001: CD001918, 2001.

9. Moghadasian MH: Cerebrotendinous xanthomatosis: Clinical course, genotypes and metabolic backgrounds. Clin Invest Med 27: 42-50, 2004.

10. Szalat R, Arnulf B, Karlin L, Rybojad M, Asli B, Malphettes M, Galicier L, Vignon-Pennamen MD, Harel S, Cordoliani F, et al: Pathogenesis and treatment of xanthomatosis associated with monoclonal gammopathy. Blood 118: 3777-3784, 2011.

11. Dagistan E, Canan A, Kizildag B and Barut AY: Multiple tendon xanthomas in patient with heterozygous familial hypercholesterolaemia: Sonographic and MRI findings. BMJ Case Rep 2013: 2013.

12. Cruz PD Jr, East $C$ and Bergstresser PR: Dermal, subcutaneous and tendon xanthomas: Diagnostic markers for specific lipoprotein disorders. J Am Acad Dermatol 19: 95-111, 1988.

13. Courtice FC: Permeability of normal and injured skin capillaries to lipoproteins in the rabbit. Aust J Exp Biol Med Sci 37: 451-463, 1959.

14. Courtice FC: The transfer of proteins and lipids from plasma to lymph in the leg of the normal and hypercholesterolaemic rabbit. J Physiol 155: 456-469, 1961.

15. Artieda M, Cenarro A, Junquera C, Lasierra $P$, Martínez-Lorenzo MJ, Pocoví M and Civeira F: Tendon xanthomas in familial hypercholesterolemia are associated with a differential inflammatory response of macrophages to oxidized LDL. FEBS Lett 579: 4503-4512, 2005.

16. Perrone G, Zagami M, Casale M, Salvinelli F, Morini S and Rabitti C: Immunohistochemistry and differential diagnosis of a solitary flat laryngeal xanthoma: A case report. In Vivo 21: 119-121, 2007.

17. Bureau NJ and Roederer G: Sonography of achilles tendon xanthomas in patients with heterozygous familial hypercholesterolemia. AJR Am J Roentgenol 171: 745-749, 1998.

18. Bude RO, Adler RS and Bassett DR: Diagnosis of achilles tendon xanthoma in patients with heterozygous familial hypercholesterolemia: MR vs. sonography. AJR Am J Roentgenol 162: 913-917, 1994.

19. Liem MS, Leuven JA, Bloem JL and Schipper J: Magnetic resonance imaging of achilles tendon xanthomas in familial hypercholesterolemia. Skeletal Radiol 21: 453-457, 1992.

20. Yamashita S, Hbujo H, Arai H, Harada-Shiba M, Matsui S, Fukushima M, Saito Y, Kita T and Matsuzawa Y: Long-term probucol treatment prevents secondary cardiovascular events: A cohort study of patients with heterozygous familial hypercholesterolemia in Japan. J Atheroscler Thromb 15: 292-303, 2008.

21. Civeira F, Castillo S, Alonso R, Meriño-Ibarra E, Cenarro A, Artied M, Martín-Fuentes P, Ros E, Pocoví M and Mata P; Spanish Familial Hypercholesterolemia Group: Tendon xanthomas in familial hypercholesterolemia are associated with cardiovascular risk independently of the low-density lipoprotein receptor gene mutation. Arterioscler Thromb Vasc Biol 25: 1960-1965, 2005.

22. Bots ML, Hoes AW, Koudstaal PJ, Hofman A and Grobbee DE: Common carotid intima-media thickness and risk of stroke and myocardial infarction: The rotterdam study. Circulation 96: 1432-1437, 1997.

23. Chambless LE, Heiss G, Folsom AR, Rosamond W, Szklo M, Sharrett AR and Clegg LX: Association of coronary heart disease incidence with carotid arterial wall thickness and major risk factors: The atherosclerosis risk in communities (ARIC) study, 1987-1993. Am J Epidemiol 146: 483-494, 1997.

24. Beigel R and Beigel Y: Homozygous familial hypercholesterolemia: long term clinical course and plasma exchange therapy for two individual patients and review of the literature. J Clin Apher 24: 219-224, 2009. 
25. Selvan JP, Uthaman B, Abushaban L and Jebaraj R: Homozygous familial hypercholesterolemia with generalized arterial disease. Med Princ Pract 16: 75-78, 2007.

26. Rader DJ, Cohen J and Hobbs HH: Monogenic hypercholesterolemia: New insights in pathogenesis and treatment. J Clin Invest 111: 1795-1803, 2003.

27. Ahn JH, Chun TJ and Lee S: Nodular excision for painful localized achilles tendon xanthomas in type II hyperlipoproteinemia: A case report. J Foot Ankle Surg 50: 603-606, 2011.
28. Moroney PJ and Besse JL: Resection of bilateral massive achilles tendon xanthomata with reconstruction using a flexor hallucis longus tendon transfer and bosworth turndown flap: A case report and literature review. Foot Ankle Surg 18: e25-e28, 2012. 29. Fahey JJ, Stark HH, Donovan WF and Drennan DB: Xanthoma of the achilles tendon. Seven cases with familial hyperbetalipoproteinemia. J Bone Joint Surg Am 55: 1197-1211, 1973. 